\title{
Low-noise Linear and Angular Motion Sensors for Vibration Monitoring
}

\author{
I.V. Egorov, D.L. Zaitsev, A.V. Neeshpapa, A.S. Shabalina
}

\begin{abstract}
*The presented researches was supported by Russian Ministry of Abstract - This article reviews on the development of a new category motion sensors for vibration monitoring including linear and angular accelerometers based on molecular electronic transducer (MET) technology. This technology utilizes liquid not only as an inertial mass but also as one of the main elements in the conversion of mechanical motion into electric current. It is possible to achieve signal amplification close to $10^{8}$. Motion sensors demonstrate wide frequency and dynamic range and sensitivity that are one to two orders of magnitude better than MEMS devices of the same size. They can provide an alternative paradigm in development of motion sensors in wide variety of applications including monitoring systems of complex engineering structures, seismic analysis of bridges and buildings, remote sensing devices, structural behavior and structure wind excitation measurement.
\end{abstract}

Keywords-instrumentation and sensing technology, motion sensor, vibrationg monitoring, accelerometers.

\section{Introduction}

Motion sensors including accelerometers and angular sensors provide measurement of movement in at least six degrees of freedom. The simplest way to do motion sensing is a solid-state mass-spring system, creating a damped simple harmonic oscillator. The movement of the solid-state proof mass can be measured with respect to displacement, velocity or acceleration by mechanical-electrical transducers. Similarly in terms of design, fabrication, and readout, accelerometers and angular measuring gyroscopes are the current leaders in commercially successful MicroElectro-Mechanical Systems (MEMS) technology. Among variety of transduction mechanisms underlying solid-state MEMS motion sensors, the most successful types are based on capacitive transduction due to the simplicity of the sensing element itself, no requirement for specific materials, low power consumption and good stability over temperature. There has been plenty of work done along the direction of scaling down the device size while maintaining low noise, high sensitivity and high resolution using MEMS techniques [1]-[3]. However, they have not yet satisfied for specific applications, especially in low frequency seismic sensing, because of the inherent limitation of working mechanism of the solid-state mass-spring system. They also have limited applicability due to the fragility to high shocks and the requirement of strict installation angle. As opposed to a solid inertial mass, a molecular electronic transducer (MET) is sensitive to the movement of a liquid electrolyte relative to fixed electrodes. The term "Molecular Electronic

I.V.Egorov, D.L. Zaitsev, A.V. Neeshpapa, A.S. Shabalina

Moscow Institute of Physics and Technology

Russia
Transducer" was introduced in Russia in the 1980s to describe such a device [4]. Inspired by the exceptionally high rate of mechanical signal conversion into electric current in MET involving mass and charge transport, pioneer studies of MET [5]-[11] provide an alternative approach in building motion sensors in wide variety of applications including monitoring systems of complex engineering structures [12]-[14], seismic analysis of bridges and buildings, remote sensing devices, structural behavior and structure wind excitation measurement.

Ultra-compact MET linear and angular motion sensors have no fragile or moving parts, high sensitivity and low noise, especially at low frequencies, and insensitivity on installation angle.

\section{Molecular-Electronic Transfer - Principle of Operation}

Fig. 1 illustrates the basic concept of MET sensing element, consisting of two transducing electrochemical cells.

An electrolyte-filled channel (1) allows the electrolyte (2) to move inertially along the channel's length. Four electrodes configured as anode(4)-cathode(5)-cathode(5)anode(4) separated by dielectric layers (3) span the width of this channel. Holes through the electrodes allow the flow of electrolyte.

Each anode-cathode pair is an electrochemical cell, in which charges are transferred between anode and cathode by ions in the electrolyte. Typically, MET uses concentrated iodine-iodide electrolyte containing potassium iodide (KI) or lithium iodide (LiI) and a small amount of elemental Iodine $\left(\mathrm{I}_{2}\right)$. The sensing mechanism of the MET is based on electrochemical cell where reversible chemical reactions transfer charge between anode and cathode via electrolyte ions in solution.

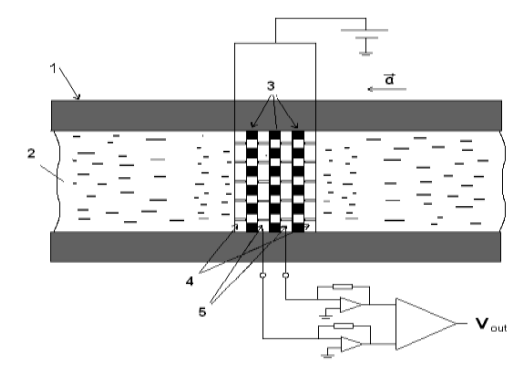

Figure 1. Schematic of the basic MET sensing element. 1 - electrolyte filled channel, 2 - electrolyte liquid, 3 - dielectric layers, 4 - anodes, 5 cathodes. 
The rate of this reaction is limited by ion concentration of the active species at the electrode's surface. [15]. In the presence of iodide, iodine turns into a soluble compound, tri-iodide, as follows [15]:

$$
I_{\mathbf{2}}+I^{-} \rightarrow I_{\mathbf{3}}^{-}
$$

When the electrodes are biased and thus current passes through the electrochemical cell, the following reversible active electrochemical reactions occur on the electrodes, on cathodes, reduction of tri-iodide:

$$
I_{3}^{-}+2 e^{-} \rightarrow 3 I^{-}
$$

on anodes, oxidation of iodide:

$$
3 I^{-}-2 e \rightarrow I_{3}^{-}
$$

Here, potassium or lithium ions $(\mathrm{K}+$ or $\mathrm{Li}+)$ play the role of the background electrolyte and do not take part in the reactions. Therefore, a distribution of active component concentration and an electrical path are established. The active charges are transferred between the anodes and cathodes in the electrolyte by diffusion, while the electrons are taken from the cathodes and transferred to the outer circuit at the anodes.

The operation principle of MET can be summarized as follows. When electric voltage is applied to the system, electrochemical current (background current) appears regardless of the presence of mechanical motion of the electrolyte. In the presence of a mechanical motion input, electrolyte starts moving due to inertia, and, besides diffusion, an additional convective transport of ions between the electrodes occurs, which leads to a significant change in the concentration gradient near the electrode surface, and therefore a change in the output current through the electrodes which in turn is determined by the concentration gradient near the electrode surface:

$$
I=-D q \oint_{S}(\nabla c, n) d S
$$

where $D$ is the diffusion coefficient, $c$ is the concentration of the charge carriers, $q$ is the charge of carriers, $\boldsymbol{n}$ is a unit vector normal to the surface of the electrode, and $S$ is the electrode surface area. Due to the fact that $I_{\mathbf{a}}^{-}$is present in a concentration far less than $\Gamma$, the cathode reaction is more sensitive to changes in ion concentration and thus, fluid flow. Therefore, difference of the cathode currents in two anode-cathode pairs can be employed as the output signal for a MET motion sensor. The output of each cell depends on the concentration gradient of electrolyte at the cathode. Although each electrochemical cell's response is non-linear with respect to fluid velocity, the combined output of both cathodes is linear for a very wide range of fluid velocities. The sensor's output current is given by [15]:

$$
I_{\text {out }}=I_{C 2}-I_{C 1}=D q\left(\oint_{S_{C 2}}(V c, \mathrm{n}) d S_{C \mathbf{2}}-\oint_{S_{C 1}}(V c, \mathrm{n}) d S_{C 1}\right)
$$

where $I_{C 1}, I_{C 2}$ are the currents through the surface of the corresponding cathodes, $S_{C 1}, S_{C 2}$ are the surface areas of the corresponding cathodes.

\section{Linear Transfer Function}

A MET motion sensor can be considered as a superposition of two systems: first, input motion is converted to fluid motion of the electrolyte by mechanical system. Next, the electrolyte's velocity is measured by the electrochemical system, resulting in an output current of the sensor. Therefore, the frequency-dependent transfer function of the entire device can be written as:

$$
H(\omega)=H_{\text {mech }}(\omega) H_{e c}(\omega),
$$

where $H_{\text {mech }}(\omega)$ describes the mechanical response of the fluidic system, which in the case of linear MET sensor, is analogous to a solid-state damped, driven harmonic oscillator. In this sense, the restoring force is provided by the rubber membranes which are located at the two ends of the channel to seal the electrolyte, and the damping force is the hydrodynamic resistance of the electrolyte as it flows through the sensing element. The equation that governs motion of the electrolyte can therefore be expressed as

$$
\frac{d^{2} V}{d t^{2}}+\frac{R_{h} S_{c h}}{\rho L} \frac{d V}{d t}+\frac{k}{\rho L} V=-S_{c h} a
$$

where $V$ is the volume of fluid passing through the channel, $a$ is the external acceleration, $R_{h}$ is the hydrodynamic resistance which is solely determined by the channel geometry in laminar flow condition, $k$ is the coefficient of volume stiffness and depends only on the characteristics of the rubber membranes, $\rho$ is the density of the electrolyte, $S_{c h}$ is the cross-section area of the channel and $L$ represents the length of the electrolyte filled channel. By transforming (5) to frequency domain, the magnitude of the transfer function of the fluid mechanical motion can be obtained as follows

$$
\left|H_{\text {mech }}(\omega)\right|=\left|\frac{Q(\omega)}{a(\omega)}\right|=\frac{\rho L}{\sqrt{\left(\frac{\rho L}{S_{c h}}\right)^{2} \frac{\left(\omega^{2}-\omega_{0}^{2}\right)^{2}}{\omega^{2}}+R_{h}^{2}}}
$$

where $Q(t)=\frac{d V(t)}{d t}$ is the volumetric flow rate, $\omega_{0}=\sqrt{ }(k / \rho L)$ is the mechanical resonance frequency of the device.

$H_{e c}(\omega)$ describes the ability of the electrochemical system to detect electrolyte motion as a function of frequency. A simple model for $H_{e c}(\omega)$ was derived analytically by Larcam [16], having a form of

$$
\left|H_{\theta e}(\omega)\right|=\left|\frac{I(\omega)}{Q(\omega)}\right|=\frac{C}{\sqrt{1+\left(\frac{\omega}{\omega_{D}}\right)^{2}}},
$$


where $C\left(\mathrm{~A} /\left(\mathrm{m}^{3} / \mathrm{s}\right)\right)$ is the conversion factor of the electrochemical cell, $\omega_{\mathrm{d}}=D / d^{2}$ is the diffusion frequency and $d$ is the inter-electrode distance.

\section{MET-Based Linear sensors with negative feedback}

The principle of operation of the molecular-electronic linear sensor containing negative feedback illustrates Fig. 2. Output signal from the sensing cell passes through the feedforward circuit $H_{f f}$ and then returns by the feedback circuit $H_{f b}$ as an electric current in feedback coil 5. Electric current in the coil, in turn, interacting with the magnet 4 fixed on the membrane 1 , generates compensation of the inertia forces developed under the influence of an external mechanical signal.

Finally, the output signal is formed by the filter $H_{f}$ which is adjusted to a necessary frequency band.

Mathematical model of the device frequency characteristics transfer function of the sensor $H_{s}$ can be described by the following expression:

$$
H_{s}=\frac{H \cdot H_{f f}}{1-H \cdot H_{f f} \cdot H_{f b}} \cdot H_{f}
$$

Assuming a deep of feedback $\left(H \cdot H_{f f} \cdot H_{f b}>1\right)$ the last expression takes the form:

$$
H_{s}=\frac{H_{f}}{H_{f b}}
$$

This model gives an expression for the transfer function independent of the transfer functions of the sensor and feedforward electronics. The example of the resulting transfer function of the linear accelerometer is shown in the Fig. 3.

The main advantages of MET-based seismic geophones and accelerometers with negative feedback lie in their extremely high coefficient of conversion of mechanical motion into an electrical signal, the possibility of a significant (compared to a traditional geophone) expansion of the frequency range both in the low- and high-frequency bands, and the simplicity and reliability of the design [17], [18]. Until recently, the instruments of this kind were used almost exclusively for measurements in the low-frequency domain. However, the developments of recent years [19] have made it possible to produce the devices with an upper operating frequency that exceeds the values typical for most accelerometers.

The experience learned from the development of such sensors indicates that the optimal operating characteristics are attained when MET-based sensors are equipped with an electrodynamic negative feedback mechanism. With a feedback, the instrument scale factor of $250 \mathrm{~V} / \mathrm{m} / \mathrm{sec}$ is achieved without using any additional amplification, which is equal to or greater than that achieved by other known lowfrequency geophones. At the same time, for a frequency range starting at $1 \mathrm{~Hz}$, the MET velocity sensor are much smaller than typical electrodynamic geophone.

MTSS-1001 is a single-axis, ultracompact, high-gain, 1$\mathrm{Hz}$ velocity sensor. MTSS-2003 contains three orthogonaloriented MTSS-1001 seismic sensors. It is probably the world's smallest tri-axis $1-\mathrm{Hz}$ seismometer. MTSS-2003 is designed primarily for measuring weak low-frequency signals. It combines high gain, small size, and low power consumption.

The 3-component high-performance accelerometer MTSS-1033A and its 1-component version MTSS-1031A are designed for strong motion seismic measurements, industrial vibration monitoring, vibration isolation analysis, etc. MET accelerometers combine very small size and high sensitivity over wide frequency band. The wide dynamic range, low distortion, high temperature stability, and competitive price make it ideal and cost-effective solution for various applications such as structural monitoring, strong motion measurements or earthquake registration. At the same time, unlike mechanical force-balanced accelerometers, the MET accelerometers are insensitive to DC component of the seismic signal i. e. gravity. Although the seismic signal is presented by non-zero frequencies, the DC signal is of significant practical importance. The DC signal makes it possible to calibrate the sensor by rotating or tilting it relative to the gravity vector and to determine the sensor's axes orientation with respect to the vertical direction. The main performance parameters for MET linear sensors are shown in Table 1.

TABLE I. THE TECHNICAL PERFORMANCE PARAMETERS FOR MET COMPACT LOW-FREQUENCY GEOPHONES AND ACCELEROMETERS

\begin{tabular}{|l|l|l|l|}
\hline \multirow{2}{*}{ Performance } & \multicolumn{3}{|c|}{ MET sensors } \\
\cline { 2 - 4 } & \multicolumn{1}{|c|}{ MTSS-1001 } & MTSS-1041A & \multicolumn{1}{c|}{ MTSS-1031A } \\
\hline Frequency range & $1-300 \mathrm{~Hz}$ & $0.1-120 \mathrm{~Hz}$ & $0.1-120 \mathrm{~Hz}$ \\
\hline Sensor noise & $\begin{array}{l}100 \mathrm{~nm} / \mathrm{sec}, \\
\text { rms over } \\
\text { frequency } \\
\text { range }\end{array}$ & $70 \mathrm{ng} / \sqrt{ } \mathrm{Hz}$ & $130 \mathrm{ng} / \sqrt{\mathrm{Hz}}$ \\
\hline Conversion factor & $250 \mathrm{~V} /(\mathrm{m} / \mathrm{sec})$ & $6 \mathrm{~V} / \mathrm{g}$ & $2.4 \mathrm{~V} / \mathrm{g}$ \\
\hline $\begin{array}{l}\text { Maximum negative } \\
\text { feedback depth }\end{array}$ & 20 & 100 & 150 \\
\hline $\begin{array}{l}\text { Maximum applied } \\
\text { acceleration }\end{array}$ & - & $\pm 2 \mathrm{~g}$ & $\pm 4 \mathrm{~g}$ \\
\hline Dynamic range & $>110 \mathrm{~dB}$ & $>110 \mathrm{~dB}$ & $>120 \mathrm{~dB}$ \\
\hline Non-linearity & $<1 \%$ & $<1 \%$ & $<1 \%$ \\
\hline Orientation & Any direction & $\begin{array}{l}\text { Any } \\
\text { direction }\end{array}$ & Any direction \\
\hline Weight & $180 \mathrm{~g}$ & $180 \mathrm{~g}$ & $180 \mathrm{~g}$ \\
\hline Temperature & $-55 \sim+65^{\circ} \mathrm{C}$ & $\begin{array}{l}-55 \sim ~ \\
{ }^{\circ} \mathrm{C}\end{array}$ & $-55 \sim+65{ }^{\circ} \mathrm{C}$ \\
\hline Power & $<80 \mathrm{~mW}$ & $<80 \mathrm{~mW}$ & $<80 \mathrm{~mW}$ \\
\hline
\end{tabular}

The forced feedback gives an opportunity to optimize the parameters of the sensor in terms of achieving maximum sensitivity and minimum self-noise level, though it is not necessary to flatten the sensor amplitude-frequency response at the feedback loop input. 


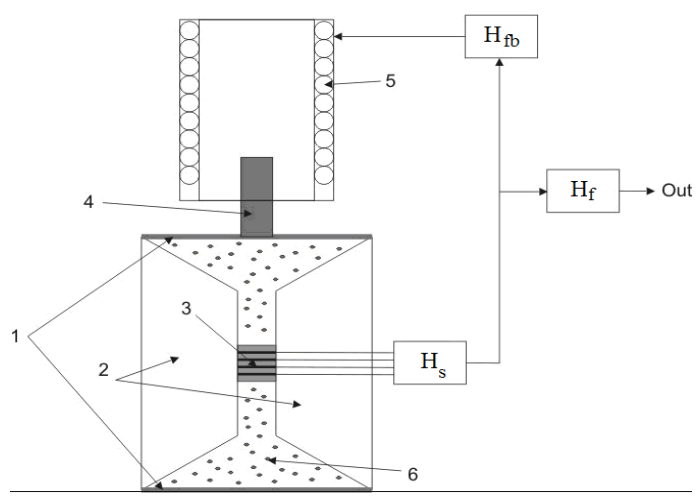

Figure 2. Sensor on the basis of MET with negative feedback. 1 - elastic membranes; 2 - sensor case; 3 - sensing cell on the basis of MET; 4 permanent magnet; 5 - feedback coil.

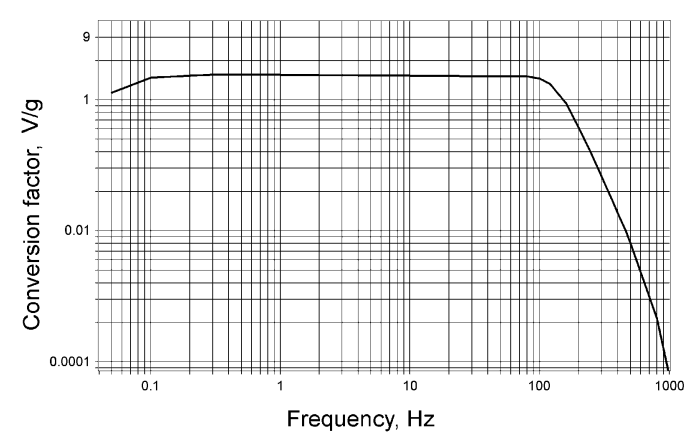

Figure 3. Transfer function of the MET accelerometere.

\section{Iv. Electrochemical Angular Motion Sensors}

The mechanical configuration of the rotational sensor, based on the electrochemical sensing technology, is presented in Fig. 4. The sensor consists of the toroidal channel, filled with an electrolyte, and the sensitive cell placed across the channel, converting liquid motion into the electrical response. An expansion volume is used to compensate for the temperature expansions of the liquid. Electrochemical sensors are probably the only rotational sensors which are commercially available at a reasonable cost with a resolution appropriate to seismic applications. The parameters of some of the commercially available electrochemical rotational seismometers are shown in Table 2. The independent test data can also be found in [20].

In accordance with the physical transduction of the mechanical effect into the liquid flow through the transductive element, molecular electronic angular motion sensors are accelerometers. All the above considered, the equation of liquid pressure in the channel of the studied angular accelerometer can be presented as follows:

$$
\dot{Q}+\frac{S_{c h} R_{h}}{2 \pi R \rho}=\mathcal{S}_{c h} R
$$

where $R$ is the toroidal channel radius, $\varepsilon$ is the angular acceleration of the accelerometer body under external effects, $Q$ is the liquid flow.

After Fourier transformation of (12), the transfer function of the mechanical system (transforming external mechanical movement i.e. angular acceleration into the liquid flow through the transducer) of the studied sensor can be easily defined:

$$
H_{\text {mech }}(\omega)=\frac{Q}{\varepsilon}=\frac{2 \pi R \rho / R_{h}}{1+i \omega / \omega_{h}}
$$

where $\omega_{h}=R_{h} S / 2 \pi R \rho$ is the hydrodynamic frequency, above which transfer function of the mechanical system reduces proportionally to $\omega^{-1}$ [21]. In realistic parameters the hydrodynamic resistance defining the value of $\omega_{h}$ for this system is so high that $H_{\text {mech }}(\omega)$ transfer function decline begins at the frequency range of tens and even hundreds of $\mathrm{Hz}$. That is why for lower frequencies mechanical transfer function will be considered a constant.

TABLE II. TECHNICAL PARAMETERS OF MOLECULAR ELECTRONIC ROTATIONAL SENSORS.

\begin{tabular}{|l|l|}
\hline \multicolumn{1}{|c|}{ Model } & \multicolumn{1}{c|}{ METR-03/01 } \\
\hline Output & Angular rate \\
\hline Sensor type & Electrochemical \\
\hline Noise at 1 Hz & $10^{-7} \mathrm{rad} / \mathrm{s} / \sqrt{ } \mathrm{Hz}$ \\
\hline Full-scale range & $0.1 \mathrm{rad} / \mathrm{s}$ \\
\hline Bandwidth & $0.033-50 \mathrm{~Hz}$ \\
\hline Size & $120 \times 120 \times 102$ \\
\hline
\end{tabular}

\section{v. Summary}

The molecular electronic sensors offer an alternative to regular electromechanical devices. They are compact, robust, and easy to install and provide reliable data. The operation principles of the molecular electronic sensors are based on using the charge transfer variations due to electrolyte motion in the four-electrode electrochemical cell. Motion sensors demonstrating wide frequency and dynamic range and sensitivity that are one to two orders of magnitude better than MEMS devices of the same size and provide an alternative approach in building the motion sensors in wide variety of applications including monitoring systems of complex engineering structures, seismic analysis of bridges and buildings, remote sensing devices, structural behavior and structure wind excitation measurement.

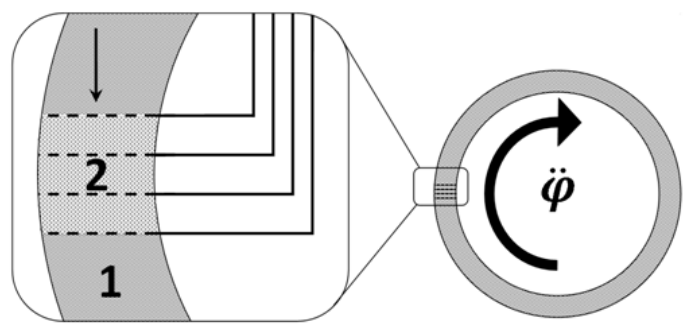

Figure 4. Molecular electronic angular motion sensor. 1 - electrolyte, 2 conversion cell. 
Proc. of the Third Intl. Conf. on Advances in Civil, Structural and Construction Engineering - CSCE 2015

Copyright (C) Institute of Research Engineers and Doctors, USA .All rights reserved.

ISBN: 978-1-63248-079-8 doi: 10.15224/ 978-1-63248-079-8-49

\section{References}

[1] W. T. Pike, I. M. Standley, W. J. Karl, S. Kumar, T. Semple, S. J. Vijendran and T. Hopf, "Design, fabrication and testing of a micromachined seismometer with nano-g resolution", Transducers 2009, Denver, CO, USA, June 21-25, 2009.

[2] F.A. Levinzon, "Ultra-Low-Noise seismic piezoelectric accelerometer with integral FET amplifier", IEEE Sensors Journal, Vol.12, No. 6, pp. 2262-2268, 2012.

[3] J. Bernstein, Raanan Miller, William Kelley and Paul Ward, "Lownoise MEMS vibration sensor for geophysical applications", Journal of Microelectromechanical Systems, Vol.8, No.4, pp. 433-438, 1999.

[4] N. S. Lidorenko, "Vvedenie v molekuliarnuyu elektroniku (Introduction to Molecular Electronics)", Moscow: Enegoatomizdat, 1984.

[5] Kozlov, V.A., A.N. Korshak, and N.V. Petkin, "Theory of diffusiontype transducers for ultralow electrolyte flow-rates", Soviet Electrochemistry, Vol. 27, No. 1, pp. 16-21, 1991.

[6] Kozlov, V.A. and M.V. Safonov, "Self-noise of molecular electronic transducers", Technical Physics, Vol. 48, No. 12, pp. 1579-1582, 2003.

[7] Kozlov, V.A. and D.A. Terent'ev, "Transfer function of a diffusion transducer at frequencies exceeding the thermodynamic frequency". Russian Journal of Electrochemistry, Vol. 39, No. 4, pp. 401-406, 2003

[8] Zakharov I.S. and V.A. Kozlov, "Stationary convective diffusion and nonlinear effects in an electrochemical transducer", Russian Journal of Electrochemistry, Vol. 39, No. 4, pp. 397-400, 2003.

[9] Panferov, A.P. and A.V. Kharlamov, "Theoretical and Experimental Study of an Electrochemical Converter of a Pulsing Electrolyte Flow". Russian Journal of Electrochemistry, Vol. 37, No. 4, pp. 394398, 2001.

[10] Reznikova, L.A. et al., "Limiting Current in Iodine-Iodide System on Vertical Electrode under Conditions of Natural Convection", Russian Journal of Electrochemistry", Vol. 37, No. 4, pp. 382-387, 2001.

[11] Kozlov, V.A. and D.A. Terent'ev, "Frequency Characteristics of a Spatially-Confined Electrochemical Cell under Conditions of Convective Diffusion", Russian Journal of Electrochemistry, Vol. 38 , No. 9, pp. 992-999, 2002.

[12] Celebi M., "Seismic Instrumentation of Buildings (With Emphasis on Federal Buildings)", Technical Report No. 0-7460-68170, United States Geological Survey, Menlo Park, CA., 2002.

[13] Yudahin F.N., G.N. Antonovskaya, N. K. Kapustian, E.V. Egorov, A. N. Klimov, "Investigation of an external impact conversion into the strained rotation inside ancient boulder structures (Solovky islands, White Sea)", 6th European Workshop on the seismic behaviour of Irregular and Complex Structures (6EWICS) O. Lavan, M. De Stefano (eds.), Haifa, Israel, 12-13 September 2011.

[14] Kapustian N., Antonovskaya G., Agafonov V., Neumoin K., Safonov M, "Seismic monitoring of linear and rotational oscillations of the multistory buildings in Moscow", 6th European Workshop on the seismic behaviour of Irregular and Complex Structures (6EWICS) O. Lavan, M. De Stefano (eds.), Haifa, Israel, 12-13 September 2011.

[15] V. G. Krishtop, V. M. Agafonov, A. S. Bugaev, "Technological principles of motion parameter transducers based on mass and charge transport in electrochemical microsystems", Russian Journal of Electrochemistry, Vol. 48, No. 7, pp. 746-755, 2012.

[16] C. W. Larcam, "Theoretical analysis of the solion polarized cathode acoustic linear transducer", J. Acous. Soc. Am., Vol. 37, pp. 664, 1965.

[17] V.M. Agafonov, I.V. Egorov, A.S. Shabalina, "Operating principles and technical characteristics of a small-sized molecular-electronic seismic sensor with negative feedback", Seismic Instruments, January 2014, Volume 50, Issue 1, pp. 1-8

[18] V.M. Agafonov, E.V.Egorov, D.L. Zaitsev, "Molecular electronic linear accelerometers. Preliminary test results", Gyroscopy and Navigation, October 2010, Volume 1, Issue 4, pp. 246-251.

[19] Krishtop V.G., Agafonov V.M., Bugaev A.S., "Technological principles of motion parameter transducers based on mass and charge transport in electrochemical Microsystems", Russian Journal of Electrochemistry, July 2012, Volume 48, Issue 7, pp. 746-755.
[20] Bernauer M., Fichtner A., Igel H., "Measurements of translation, rotation and strain: new approaches to seismic processing and inversion", Journal of Seismomlogy, V. 16, Issue 4, pp. 669-681.

[21] R. Leugoud, A. Kharlamov, "Second generation of a rotational electrochemical seismometer using magnetohydrodynamic", Journal of Seismomlogy, 2012, V. 16, pp. 587-593.

About Authors:

Research Physicist of the Center
for Molecular Electronics Moscow
Institute of Physics and
Technology.

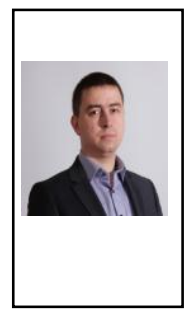

Ph.D., Senior Research Physicist of the Center for Molecular

Electronics Moscow Institute of Physics and Technology.

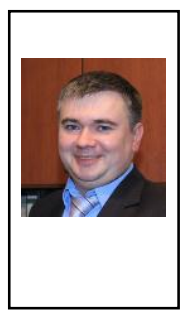

Research Physicist of the Center for Molecular Electronics Moscow Institute of Physics and Technology.

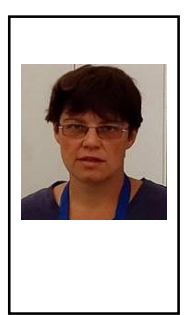

Research Physicist of the Center for Molecular Electronics Moscow Institute of Physics and Technology. 